\title{
A View-based Analysis of Distributed and Mobile Teams
}

\author{
Christoph Dorn, Daniel Schall, Robert Gombotz, Schahram Dustdar \\ VitaLab, Distributed Systems Group, Institute of Information Systems \\ Vienna University of Technology,Vienna, Austria \\ Email: \{dorn|schall|gombotz|dustdar\}@infosys.tuwien.ac.at
}

\begin{abstract}
In this paper, we present emerging team forms, which are classified into Nimble, Virtual, and Mobile teams. Based on this classification, we present a systematic analysis of specific team characteristics at different views to outline the idiosyncrasies of these heterogeneous team forms. These views are domain independent and highlight team features that can be found in every team however to a varying degree. The five views - Spatial, Organizational, Project, Human Interaction, and Service - help to group related team attributes and serve as a basis for a requirements analysis in collaborative working environments. The derived model places such requirements into four categories: Team Management, Team Interactions, Information in Teams, and Technology Requirements.

The inContext platform (part of the European funded research project inContext) accounts for these idiosyncrasies and requirements by applying a serviceoriented approach.
\end{abstract}

\section{Introduction}

Globally dispersed companies, rapidly changing requirements, shifting markets, and increasing competition are just a few factors that shape current collaborative working environments (CWE). The recent transformation from traditional team structures towards distributed, cross-organizational, and highlyflexible collaborative working environments calls for adaptive tools and services to provide effective and efficient support to e-professionals.

An analysis of team characteristics and requirements is pertinent to understand these new collaborative environments. Only then we will be able to target a specific user group more precisely and to ultimately create adaptive services. Moreover, a better understanding of team idiosyncrasies will allow us to compare and evaluate existing and future approaches, tools, and architectures.

Previous research $[4,11]$ indicates the need for classifying emerging teams according to three forms Nimble, Virtual, and Nomadic (N/V/M). Scenarios from our partners in the EU STREP project inContext support this categorization.

To this point, past research focused on specific concerns such as communication support [6], collaboration support [10], or team awareness support [5] thereby neglecting the overall team and collaboration characteristics. In contrast, research efforts aiming to cover multiple issues [7] focused rather on tool integration rather than on tool adaptation.

In addition, several scientific studies discussed team performance $[1,8]$, management and leadership issues $[2,12]$, communication as well as organizational challenges [9], but a fundamental analysis of emerging team characteristics received little attention. A related concept is the notion of Virtual Communities from which Virtual Teams [3] arise. However, here the focus is on the community aspect rather than on the different team requirements.

We will briefly revisit emerging team forms which serve as basis for our team characteristics model. This in turn provides the required input for the analysis of team form requirements. We conclude this paper by outlining some future work and trends.

\section{Emerging team forms}

Based on various criteria such as team goal, coupling, time span, etc., we classify emerging team forms into Nimble, Virtual, and Mobile teams (N/V/M teams). A nimble team quickly gathers to work on problems that may emerge unexpectedly. Team members can be distributed or collocated in terms of physical space. Team leadership is established in an ad-hoc fashion, whilst peers may take up multiple roles simultaneously. Examples for nimble teams are task forces of specialists for crisis mitigation in healthcare 
(e.g. SARS) or scientists organizing a conference at a new location.

Virtual team members collaborate across geographical distance and organizational boundaries and have a somewhat stable team configuration with roles and responsibilities assigned to the team members. Exemplary virtual teams are technical consultants for a mechanical engineering project or a production team for a movie.

Members of nomadic teams are typically involved in several projects at the same time in a loosely coupled fashion. As the name suggests, the concept and model of mobile teams aims to characterize and support team members that are highly mobile and frequently change their location and move to different places where they may meet other collaborators. Collocation of peers, without being explicitly planned or scheduled, yields the need to opportunistically collaborate by exchanging data and artifacts in an adhoc fashion. Experts in a political conflict resolution, musicians providing a composition of soundtracks, or actors providing stunt or dubbing services are some real-world examples.

Table 1 shows significant features of these team forms:

Table 1: Emerging team forms

\begin{tabular}{|l|l|l|l|}
\hline & Nimble & Virtual & Nomadic \\
\hline Vision \& Goals & $\begin{array}{l}\text { Strongly } \\
\text { Shared }\end{array}$ & Shared & $\begin{array}{l}\text { Not } \\
\text { Shared }\end{array}$ \\
\hline Team Coupling & Tight & Loose & None \\
\hline $\begin{array}{l}\text { Time Span of } \\
\text { Existence }\end{array}$ & $\begin{array}{l}\text { Short- } \\
\text { lived }\end{array}$ & $\begin{array}{l}\text { Project- } \\
\text { dependent }\end{array}$ & $\begin{array}{l}\text { Not } \\
\text { known }\end{array}$ \\
\hline $\begin{array}{l}\text { Team } \\
\text { Configuration }\end{array}$ & Flexible & Stable & Dynamic \\
\hline Team Size & $\begin{array}{l}\text { Compact } \\
\text { (ca. 10) }\end{array}$ & $\begin{array}{l}\text { Large } \\
\text { (ca. 50) }\end{array}$ & Large \\
\hline
\end{tabular}

To better understand these team forms and their implications we need to elaborate on a number of characteristics. Combining related characteristics into a logical view (e.g., spatial view) improves clarity and by connecting these views we can derive a team characteristics model. Views can be used to analyze the effects and interdependencies of characteristics within team forms including those that have intermediary and fuzzy forms, as well as transitions between those forms.

\section{Team characteristics}

We have established a Team Characteristics Model that basically decomposes characteristics into views (i.e., the type of context that plays a role for teams), temporal aspects such as setup phase, actual collaboration, and termination of collaboration, for example termination of a team, and finally the specific team characteristics as a dimension in this model. The model is shown in Figure 1.

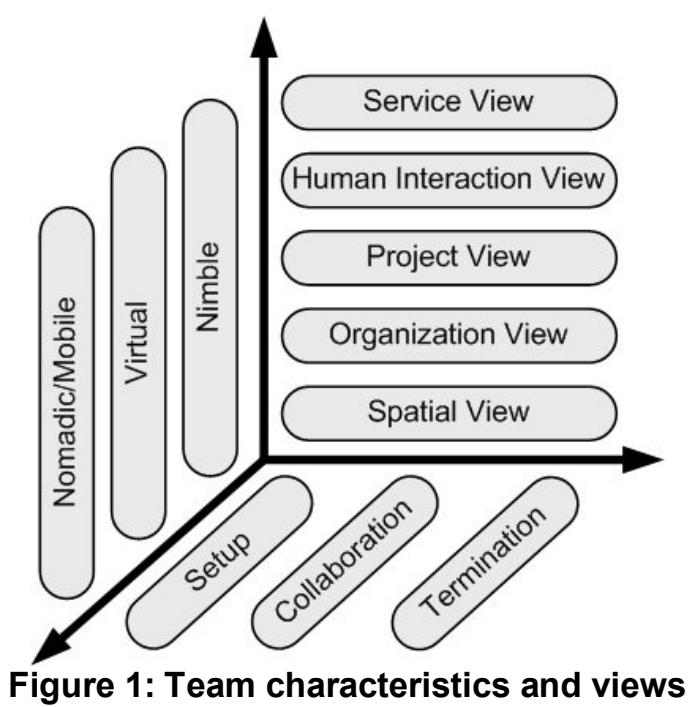

\subsection{Spatial view}

Physical space can be characterized by geometric quantities such as volume, area, point, and extension of space. We look at measures that characterize entities such as users within space and their relation to one another. A position in space is expressed by location information that can also provide a semantic description or representation of space. The spatial distribution of team members is the geographical distance of individuals as well as distance between sub-teams. From a team's point of view, a proximity measure may be used to indicate distance or distribution. Temporal aspects such as transition of entities to a new location or frequency of location changes are expressed by location dynamics. Dynamics applied to teams include information such as the likelihood of intersecting trajectories or variations due to entities traveling at different speed. Awareness of location information and dynamics is a prime concern of the different team forms. Being aware of location is not only a question of observing the location context of other entities, but also the ability to recognize places or discover situated entities such as infrastructure elements and computing devices.

Significant characteristics in this view include:

- Distribution of Team Members

- Location Representation

- Location Awareness

- Location Dynamics

- A-Priori Infrastructure Knowledge 


\subsection{Organization view}

The organizational level defines the structure of an organization. Specifically, it defines a topology, which denotes links and relationships between workers, employees, and departments. Staff members take up different (organizational) roles within organizations/departments such as supervisor, manager, and CEO. Relationships and roles are essential for trust building among individuals as well as between sub-divisions. An organization may define rules and guidelines at different levels in the topology that have impact on for instance how people communicate and execute projects in different team forms. These rules can be manifested in form of policies and are referred to as enterprise culture.

Following properties denote the significant differences at the organizational level in N/V/M teams:

- Organizational Topology

- Trust

- Supervisor or Supervision

- Enterprise Culture (e.g., enterprise specific policies)

\subsection{Project view}

The project view aims at organizing and managing resources. It includes the definition of scope of required work (project goals) and planning and monitoring of constraints such as time, cost, and risk. Projects are usually instantiated under a certain premise, for instance a company's mission, or the mission and goals of nimble teams. The mission is shaped by the management unit and team leaders. The responsibility of a leader is to ensure that the actual outcome is created such that defined constraints and desired quality are satisfied, i.e., by monitoring and coordinating project related activities. People exercise various roles in a project. We note that people increasingly work on more than one project or set of activities simultaneously. The role might depend on a particular task in a certain project (peer membership and cohesion of teams).

The significant properties are:

- Leadership Establishment

- Project Task Topology

- Communication Topology

- Coordination Needs

- Risk Management

- Goal Definition

\subsection{Human interactions and patterns}

Interaction patterns are activities or activity steps that are frequently repeated and can be observed by means of pattern detection between human actors. Such patterns may impact a team, in terms of being aware of dynamics, status updates, etc., as well as the measures that can be taken to support collaboration. A communication pattern in human collaboration shows how distributed teams exchange information by means of synchronous or asynchronous communication channels. Whether or not individual interactions have great or only limited impact on a team is limited by the scope of an interaction.

Significant characteristics in this view include:

- Interaction Patterns

- Team Roles

- Ratio of Coordination versus Collaboration

- Communication Types

- Scope of Interactions

\subsection{Service interactions}

Services are means for supporting the user/teams in project dependent activities and tasks. Service interactions are related to situations that arise when services engage in concurrent and interrelated interactions. Services may be consumed in combination by sequential, parallel or conditional execution of tasks. Patterns provide the foundation for aggregating a number of services that are used in combination. Providing a service from a pool of available services, considering the consumer's context, is defined as relevance based service-provisioning. A service is provided upon request - reactive - or provided based on context - pro-actively, thereby considering collaborative activities or a task at hand. By understanding service interactions, a set of aggregated services could be provided so that human collaborators are able to complete an activity or progress towards an objective.

With respect to service interactions, important aspects for N/V/M teams are:

- Search and Discovery

- Aggregation Patterns and Automatic Provisioning

- Reliability and Availability

\subsection{Cross-cutting concerns}

These five views may be analyzed independently or a "cross-layer" approach may be taken. For example, a spatial to organizational view mapping describes where members of an organization are located. Similarly, an 
organization to project mapping links team members to their organizational background. A mapping from the project view to the human interaction view indicates which members engage in what type of interaction. Based on different roles in a project and human interactions we may observe patterns such as delegate work items, an interaction that may denote a Broker pattern. At the top-most levels, human interaction to service mapping describes what and how services are employed to achieve the goals as defined in the project.

\subsection{Impact of team views on the development of CWEs}

As stated before, characteristics within a given view apply to every team form, but have distinct and specific properties as well as impact depending on the team form in question.

Nimble teams are mostly affected by the project and human interaction view due to the tight coupling and consequent high collaboration need. In contrast, nomadic teams are heavily influenced by spatial and services issues due to their mobility and the need to work anytime and from anywhere. Virtual teams are greatly challenged by organizational, human interaction and project characteristics as such teams exist across organizational boundaries.

Keeping these distinctive team features in mind, it becomes apparent that the established team forms, $\mathrm{N} / \mathrm{V} / \mathrm{M}$, need to be supported in different ways.

Another aspect of teams is the possibility of team transitions or changes in the configuration, meaning that a team's properties (e.g., team size or coupling) resemble some other team form best. As an example, some members of a virtual team might be increasingly engaged in projects that require them to work while being mobile. Thus, these team members can be considered as part of a mobile or nomadic team.

These changes in the team configuration will have impact on the services being used by or provisioned to the team. Services need to adapt to those changes autonomously.

\section{Team requirements}

The CWE domain comprises several research areas, such as team awareness, collaboration (activity management, leadership, team performance, and conflict resolution), human interactions and patterns, context, group dynamics and human behavior, and technology aspects such as communications and mobility. Thus, as shown in Figure 2, we structure requirements in four segments, Management, Interaction, Information, and Technology.
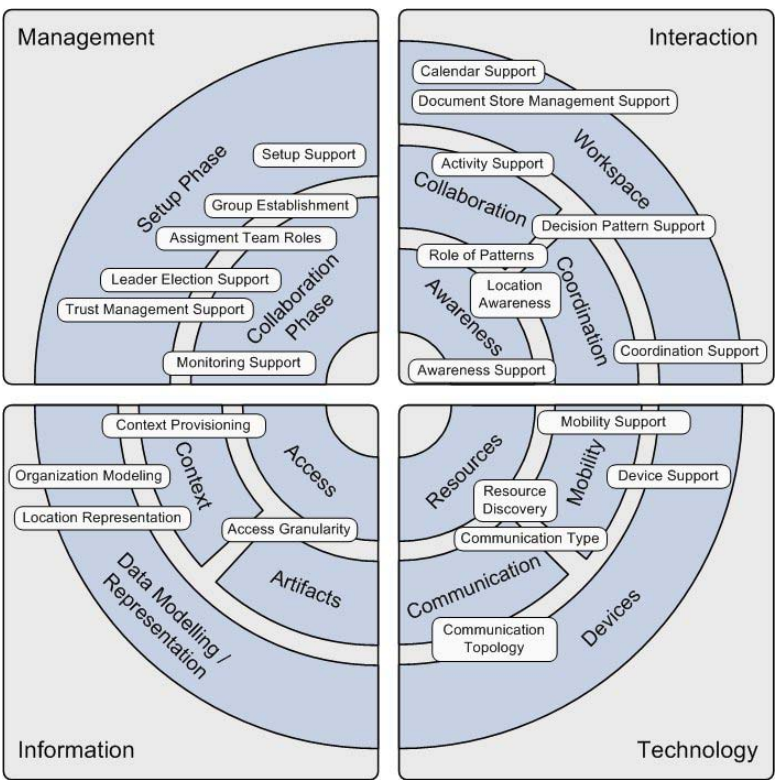

Figure 2: Team requirements

\subsection{Team management}

Management in teams with varying degree of coupling and lifetime can be explained by looking at the essential collaboration lifecycle steps, the setup, collaboration, and termination phase. Considering team characteristics in establishment of team structures and management of control flows fosters productivity and minimizes risks associated with (globally) dispersed operating teams.

Setup Support focuses on the required functions regarding non-human resources during team setup. Complementary, Group Establishment discusses factors concerning peer member ship and group establishment. One step further, Assignment of Team Roles points out the need for assignment of different roles and the required support for role dynamics. One major role is the team leader. Hence, an appropriate election mechanism is required as presented by in Leader Election Support. Upon team establishment, peers implicitly (on a social level) and explicitly (on a service level) apply trust during their interaction with others. Trust Management Support expresses the need for different techniques for the three team forms. Once team work has begun, stakeholders require an adequate Monitoring Support to keep an overview of team status and work progress.

\subsection{Team interactions}

In collaborative environments, human interaction patterns represent periodic activities describing the 
sequence and type of situation dependent interactions between individuals. Unveiling such patterns helps in optimizing collaborative activities that are executed by utilizing collaboration tools and services, and, furthermore, information and coordination flows between humans. Various software services are provided to enable team collaboration, the information that is being exchanged is adapted based on interaction patterns, and team awareness is raised.

Interaction principles and requirements can be classified according to their impact on Awareness, Collaboration, Coordination, and Workspace. Workspace is basically a high level term for standard services that are usually supported in any collaboration domain. For example, Calendar Support identifies the need for basic calendar features that may include automatic constraint checking (i.e., team calendar), synchronization between calendars, etc. Document Store Management Support is mainly concerned with the features a document management service needs to provide such as example versioning. Activity Support describes on a higher level how collaboration is conducted. Activities describe the user actions in collaboration. If activities are shared with others, monitoring task such as what is the status of activity $\mathrm{X}$ and coordination can be realized (e.g., Coordination Support through activities). At an abstract level, the Role of Patterns describes how interaction patterns are mined to improve collaboration, coordination, and awareness. Another way to raise awareness is through location information (i.e., Location Awareness). It deals with the question what kind of location information is most useful for the various team forms.

Awareness Support is the combination or unification of a number of context elements such as location, availability, presence, and activity.

\subsection{Information in teams}

Information in teams is the collection of data that is accessible by or exposed to individuals. Those data comprise contents developed by teams undertaking collaborative activities or other data that helps people to coordinate activities and recognize situations. Awareness of situations such as work, people, and places is established by means of context that has to be presented in a suitable way. Access to shared artifacts and, in addition, the way context is presented is shaped by the specific team needs.

Information principles and requirements determine Data Modeling \& Representation, Context, Artifacts, and respective Access issues. Context Provisioning requirements determined how context needs to be modeled for different team forms, how it is stored and processed and also how it is retrieved. As a form of context, Organization Modeling describes the need for making the organizational background of team members and related rules and behavior explicit. As location reveals a plethora of contextual information, Location Representation points out the need for different ways of modeling such context. Finally, Access Granularity emphasizes the necessity for different access methods for both context information and artifacts.

\subsection{Technology requirements}

Technology mediates (human) interactions and facilitates collaboration in distributed as well as collocated teams. The key point is to provide technologies that assist humans in ad-hoc collaborative activities and to recognize these activity-based interactions to establish awareness and to track progress. Employed technologies need to be able to switch from ad-hoc mode to structured mode, as well as to support nomadic peers, enabling homogeneous and heterogeneous teams to work effectively.

Technology principles and requirements cover Devices, Communication, Mobility, and Resources. Peers required various degrees of Mobility Support depending on their team form and role within the team. In doing so, they use a heterogeneous set of devices sequentially or concurrently, thus demanding for Device Support. Such mobile team members require intelligent Resource Discovery methods to reduce time-to-work. When it comes to collaboration and coordination, the Communication Type determines whether peers communicate synchronously or asynchronously and what rich media types are applied. Finally, dedicated support of the prevailing Communication Topology within each team form is needed.

\section{Discussion and Future work}

The presented model provides the theoretical foundation for three major challenges in supporting emerging team forms.

As foundation for classifying teams, the model defines the significant indicators. Our next research steps will addresses an automatic classification mechanism through reasoning on our team form-based context model.

Once classified, collaboration services can adapt themselves according to the identified requirements. Such services are central to the Pervasive Collaboration Service Architecture (PCSA), developed in the EU STREP project inContext <http://www.incontext.eu>. 
Finally, the model provides the guidance for detecting (again within the context model) team transformations respectively individual work context switches to trigger autonomic service reconfigurations.

Future work will consist of implementing the context model and reasoning algorithms as well as selfadapting collaboration services. At the same time we intend to continually increase the number of requirements and fine-tune the team form characteristics.

\section{Conclusions}

Based on our previously identified team forms, we presented a team characteristics model consisting of a spatial, organizational, project, interaction, and service view. This model not only allows discussing team forms and their transformations but also outlines aspects found most significant for a collaborative context model.

Our team requirements model serves as a framework for identifying the different requirements in collaborative working environments.

Outlining the major aspects of emerging team forms, their interdependencies and requirements we conclude that a dynamic, adaptive, service-oriented architecture is imperative to realize adequate tool support.

\section{Acknowledgements}

Part of this work was supported in part by the EU STREP Project inContext (FP6-034718) and Austrian Research Fund (FWF) Project OMNIS.

\section{References}

[1] P. Balthazard, R.E. Potter, and J. Warren, "Expertise, extraversion and group interaction styles as performance indicators in virtual teams: how do perceptions of IT's performance get formed?", SIGMIS Database 35, 1, ACM Press, 2004, pp. 41-64.

[2] W.F. Cascio; and S. Shurygailo, "E-Leadership and Virtual Teams", Organizational Dynamics, 31(4), Elsevier Science, 2003, pp. 362-376(15).

[3] S. Crave and S. Ladame "Virtual Teams and Virtual Organizations", Proceedings of the ICT Knowledge Society and Changes in Work conference (CSWO), Thematic Workshop on Virtual Communities, 2005

[4] Dustdar, S., "Caramba - A Process-Aware Collaboration System Supporting Ad Hoc and Collaborative Processes in Virtual Teams", Distributed and Parallel Databases, 15(1), Springer, 2004, pp. 45-66
[5] A. Ferscha, "Workspace awareness in mobile virtual teams", Proceedings of 9th International Workshops on Enabling Technologies: Infrastructure for Collaborative Enterprises (WETICE), IEEE Press, 2000, pp. 272-277.

[6] K. Pripuzic, L. Gjenero, and H. Belani, "Improving Virtual Team Communication", Proceedings of the International Conference on Software in Telecommunications and Computer Networks (SoftCOM), 2006, p. 266-270

[7] S.F. Ochoa, and R. Aldunate, "Collaboration Services to Support Interactions in Disaster Relief Scenarios ", Proceeding of the 8th International Conference Advanced Communication Technology (ICACT), Vol.2, 2006, pp. 12251230.

[8] D.S. Staples, and A.F. Cameron, "The Effect of Task Design, Team Characteristics, Organizational Context and Team Processes on the Performance and Attitudes of Virtual Team Members", Proceedings of the 38th Annual Hawaii international Conference on System Sciences. HICSS'05, IEEE Computer Society, 2005.

[9] J. Suchan, and G. Hayzak, "The communication characteristics of virtual teams: a case study", Professional Communication, IEEE Transactions, 44(3), IEEE Press, 2001, pp. 174-186.

[10] C. Sun, S. Xia, D. Sun, D. Chen, H. Shen, and W. Cai, "Transparent adaptation of single-user applications for multiuser real-time collaboration". ACM Trans. Computer.-Hum. Interact., 13(4), ACM Press, 2006, pp. 531-582.

[11] D. van Thanh, I. Jørstad, and S. Dustdar, "Mobile Multimedia Collaborative Services". In Handbook of Research on Mobile Multimedia, Edited by Ismail Khalil Ibrahim, Idea Group Publishing, USA, 2006.

[12] S. Zhang, J. Fjermestad, and M. Tremaine, "Leadership Styles in Virtual Team Context: Limitations, Solutions and Propositions", Proceedings of the 38th Annual Hawaii international Conference on System Sciences. HICSS'05, IEEE Computer Society, 2005. 